\title{
Erasme et le célibat sacerdotal
}

\section{Léon Ernest Halkin}

\section{Résumé}

Devant la crise du sacerdoce, Erasme adopte une position originale et généreuse. Il souhaite que le mariage soit permis aux prêtres qui ne peuvent supporter le célibat tout en désirant poursuivre leur mission. C'est de l'Eglise qu'il attend une politique d'indulgente compréhension, tandis qu'il désapprouve les unions contractées en dépit des lois ecclésiastiques par les prêtres dissidents. Pour lui-même, il ne demande rien : son indépendance lui suffit. Toutefois, il n'est pas un marginal du sacerdoce et son dernier livre, l'« Ecclesiastes », est essentiellement destiné à ses confrères du clergé paroissial.

\section{Citer ce document / Cite this document :}

Halkin Léon Ernest. Erasme et le célibat sacerdotal. In: Revue d'histoire et de philosophie religieuses, 57e année n4,1977. pp. 497-511;

doi : https://doi.org/10.3406/rhpr.1977.4405

https://www.persee.fr/doc/rhpr_0035-2403_1977_num_57_4_4405

Fichier pdf généré le 02/09/2020 


\section{ÉRASME ET LE CÉLIBAT SACERDOTAL}

SOMMAIRE :

Devant la crise du sacerdoce, Erasme adopte une position originale et généreuse. Il souhaite que le mariage soit permis aux prêtres qui ne peuvent supporter le célibat tout en désirant poursuivre leur mission. C'est de l'Eglise qu'il attend une politique d'indulgente compréhension, tandis qu'il désapprouve les unions contractées en dépit des lois ecclésiastiques par les prêtres dissidents. Pour lui-même, il ne demande rien: son indépendance lui suffit. Toutefois, il n'est pas un marginal du sacerdoce et son dernier livre, l'a Ecclesiastes $\triangleright$, est essentiellement destiné à ses confrères du clergé paroissial.

A François WENDEL, en souvenir et en hommage.

Le célibat sacerdotal est un de ces problèmes qui accompagnent le christianisme à travers sa longue histoire. Les prêtres seront-ils astreints à la continence perpétuelle ? Tout est là !

Erasme de Rotterdam (1469-1536) s'est déclaré, à maintes reprises, favorable au mariage des prêtres, mais la libération qu'il souhaite est soumise à plusieurs conditions dont l'importance n'est pas petite, comme nous allons le voir.

Ce qu'il demandera pour d'autres, Erasme le refuse pour lui-même. In n'a jamais renié ni oublié son sacerdoce, alors qu'il a rompu allè- 
grement avec la vie conventuelle. On pourrait dire de lui qu'il a a épousé la Parole ». Il a vécu, durant la plus grande partie de son existence, comme un prêtre libre, évitant tout scandale et se consacrant à ses études.

Deux historiens se sont particulièrement intéressés à la pensée d'Erasme et à ses réactions en ce domaine. E.-V. Telle a consacré un gros volume à Erasme de Rotterdam et le septième sacrement ${ }^{1}$. J. Coppens est l'auteur d'une étude critique intitulée Erasme et le célibat ${ }^{2}$. Tous deux, - le premier surtout, - sont assez peu favorables aux idées religieuses d'Erasme, mais ils connaissent parfaitement son évolution intellectuelle.

Aujourd'hui, nous rouvrons ce dossier en nous reportant sans cesse aux œuvres d'Erasme, - livres de théologie ou justifications polémiques $^{3}$, - sans négliger son énorme correspondance ${ }^{4}$, qui nous donne presque au jour le jour les sentiments d'un prêtre humaniste sur les problèmes de son temps.

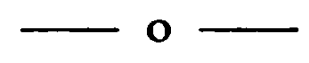

L'Encomium matrimonii ${ }^{5}$, publié à Louvain en 1518 , a sans doute été rédigé bien plus tôt, peut-être même avant 1500 . Tout au long de cette declamatio, - une œuvre mineure d'une importance majeure ${ }^{6}$, Erasme s'intéresse au célibat ecclésiastique presque autant qu'au mariage parce que, dans la problématique de son temps, mariage et célibat sont des termes corrélatifs, sinon opposés. Il veut démontrer que la vertu est possible dans le mariage, - parfois sous-estimé par certains théologiens, - alors que le vœu de chasteté, malgré le pieux halo qui l'entoure, s'accommode trop souvent de transgressions graves.

1 . V. Telle, Erasme et le septième sacrement. tuae a'évangélisme matrimoniai au XVIe siecle et contribution à la biographie intellectuelle d'Erasme, 500 p. In-80, Genève, 1954 - Du même auteur : Erasmus Roterodamus. Dilutio eorum quae Iodocus Clithoveus scripsit adversus declamationem Des. Erasmi Roterodami suasoriam matrimonii, 106 p. in-8 ${ }^{\circ}$, Paris, 1968. Nous citerons cet ouvrage sous le titre abrégé : Dilutio.

2 J. Coppens, Erasme et le célibat, dans l'ouvrage collectif, edité par le même auteur, Sacerdoce et célibat, p. 443-458, Louvain, 1971. - Du même auteur : In epistolam Des. Erasmi Roterodami de delectu ciborum ad Christophorum, episcopum Basiliensem. Scholia eiusdem defensoria, dans l'ouvrage collectif, edité par le même auteur, Scrinium Erasmianum, D. 599-620, Leyde, 1969. Nous citerons cet ouvrage sous le titre abrégé : Scholia.

3 Crasme, Opera omnia (éd. J. Clericus), 10 tomes en 11 vol. 1n-1ol., Leyde, 1703-1706. - Grasme, Opera Omnia (éd. internationale), 8 voi. in-4 parus, Amsterdam, 1969-1977. Nous citerons ces deux collections par leurs sigles habituels : L.B. (Leyde) et A.S.D. (Amsterdam).

4 P. S. Allen, Opus epistolarum Desiderii Erasmi Roterodami, 12 vol. In-8॰ (dont un vol. d'index), Oxford, 1906-1958. Nous citerons cet ouvrage sous ie titre abrégé : Opus.

5 A.S.D., t. I-5, p. 333-416. - Republié en 1522, avec d'importantes additions, dans le $D e$ conscribendis epistolis (A.S.D., t. I-2, p. 400-429), sous le titre peu explicite de Exemplum epistolae suasoriae. - Voir J. Coppens, Erasme et le cellbat, dans sacerdoce et célibat, p. 446 sv. Louvain, 1971.

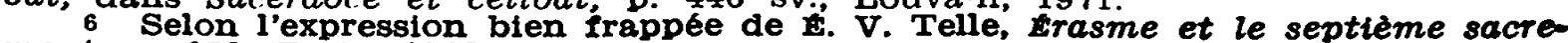
ment, p. 158, Paris, 1968. 
Après avoir exalté le mariage en général, Erasme n'hésite pas à traiter du mariage des prêtres. Il y est favorable, sous certaines conditions, afin de parer au péril causé par les prêtres concubinaires, dont le mariage régulariserait enfin la situation: " Il paraît sage de remédier de notre mieux à l'état réel des mœurs en accordant, dans la mesure du possible, le droit de se marier aux prêtres, ainsi qu'aux moines, d'autant que le nombre des prêtres est immense partout, alors que si peu mènent une existence chaste. Il est souhaitable de transformer en épouses les concubines. Ainsi les prêtres pourront, à la vue de tous et avec honneur, partager la vie de ces femmes qui leur donnent aujourd'hui mauvaise réputation et mauvaise conscience. Ils en auront des enfants qu'ils aimeront comme on aime ses enfants légitimes, des enfants qu'ils éduqueront saintement, dont ils n'auront pas honte et qui les respecteront. Je crois même que les officiaux des évêques auraient déjà veillé à cela s'ils n'escomptaient plus de profit des concubines que des épouses. ¿ ${ }^{7}$

La fin de ce texte insiste sur un double scandale, celui des prêtres fornicateurs d'abord, ensuite celui des officiaux et des évêques qui tirent avantage de la fornication des prêtres ${ }^{8}$.

Sur ces points, aucun doute n'est possible, - encore que les abus ne puissent être mesurés avec une précision statistique, - tant sont nombreux les témoignages des contemporains au sujet de l'immoralité ecclésiastique $^{9}$ : trop de prêtres menaient alors une vie conjugale à peine clandestine ${ }^{10}$. L'existence d'une taxe perçue par les juges ecclésiastiques, à titre d'amende, sur les concubinaires illustre l'étendue du mal. Erasme n'est pas le premier à dénoncer cette taxe ${ }^{11}$. Il la condamne

7 A.S.D., t. I-5, p. 402-404, 1. 224-226. En 1522, frasme a ajouté tout ce qui suit les mots " ainsi qu'aux moines ": A.S.D. t. I-2, p. 418, 1. 3-10. Voir i. V. Telle, Erasme et le septieme sacrement, p. $168 ; 1$ Idem, Dilutio, p. 29, 83. Voir auss 1 Supputationes, dans $L . B$ t. IX, col. 488 F -489 A. D Dans ce docusuite, à ne parler que des prêtres, sans plus. Le cas des moines est aussí grave que celui des séculiers, mais leur mariage pase plus de problèmes. Voir cependant la note 54 .

8 On voit combien les fondements historiques de la problématique d'rasme diffèrent de ceux des prêtres qui demandent aujourd'hui à l'tglise le droit de se marier. Cfr J. Lynch, Critique de la loi du celibat dans l'Église catholique, dans Concilium, no 78, p. 53-68, Paris, 1972.

9 On sait qu'erasme iui-même était le flls d'un prêtre. - Les Statuts synodaux reprenaient régulièrement les mêmes interdictions. Les tribunaux ecclésiastiques réprimaient les abus les plus voyants. - Même un ennemi d'trasme comme Josse Clichtove devait reconnaitre la banallté du falt: Propugnaculum Ecclesiae, p. 348, 349, 355, 374, Cologne, 1526; cfr J.-P. Massaut, Josse Clichtove, l'humanisme et la réforme du clergé, t. II. b. 204-207, Paris, 1968.

10 H. Tưchle etc., Nouvelle histoire de l'Eglise, t. III, p. 34, Paris, 1968. tisé (comme les conciles antérieurs et postérieurs) le concubinage des prêtres, ont explicitement condamné le principe d'une taxe à prélever sur les concubinalres. Cfr G. Denzler, Grunalinien der Zölibatgeschichte, vom Constanciense bis zum Tridentinum (1414-1545), dans l'ouvrage collectlf Vón Konstanz nach Trient, p. 347, Munich, 1972. Volr aussi H. C. Lea, History of sacerdotal celibacy, $3 \mathrm{e}$ ed., t. II, p. 55, Londres, 1907. - Hefele-Leclercq, Histoire des conciles, t. vIII, p. 496, Paris, 1921. - G. Pfeilschifter, Acta Reformationis Catholicae, t. I, p. 481, Ratisbonne, 1959 - A. Franzen, Zölibat und Priestereihe, 3e éd., p. 97, n. 32, Munster,

1971. T. Telle, Erasme et le septième sacrement, p. 195, 196. - L Massaut, op. cit.,
t. II, p. 
plus sévèrement que l'immoralité qu'elle consacre ${ }^{12}$. Il y reviendra en 1522 : Si episcopi tentent mutare, fortasse reclament officiales qui plus sentiunt redituum ex concubinis sacerdotum quam sensuri sint ex uxoribus ${ }^{13}$. Par ailleurs, jamais Erasme n'appellera la violence au service de la morale : il ne veut pas, comme Jacques Wimpfeling à la même époque, traquer les concubinaires ${ }^{14}$.

Dans ses Annotationes in Novum Testamentum, Erasme revient à différentes reprises sur cette question délicate. Commentant la Première Epître à Timothée (III, 2) sur les qualités requises de l'épiscope, qui ne doit être marié qu'une seule fois, il remarque que le célibat ecclésiastique est devenu un fardeau pesant pour beaucoup : «On jugera sans doute préférable d'accorder le droit de se marier publiquement à ceux qui ne peuvent absolument pas observer la continence afin qu'ils puissent vivre purement et saintement, sans être mal jugés, au lieu de se livrer à la débauche pour leur malheur et pour leur honte. ${ }^{15}$ Et il ose ajouter : a Je crains qu'à présent les impôts ecclésiastiques ne châtrent les clercs plus efficacement que le souci de la vertu. ${ }^{16}$

La Première aux Corinthiens (VII, 1-10) lui inspire des observations du même ordre. Reprenant les mots de saint Paul (si non se continent), il les applique aux prêtres de son temps (qui non continent) pour leur souhaiter plutôt sanctum connubium et immaculatum torum ${ }^{13}$.

Nous lisons dans le commentaire de l'Evangile de saint Matthieu (XIX, 12) les affirmations les plus dures et les jugements les plus tranchants sur le célibat ecclésiastique. Qui sont ceux qui se font eunuques propter regnum coelorum? Erasme répond audacieusement : "Dans ce groupe, nous plaçons ceux qui, par tricherie ou par crainte, ont été forcés au célibat, de sorte qu'il leur est permis de forniquer, mais non de se marier. Ont-ils une concubine? Ils passent pour prêtres catholiques, mais s'ils préféraient avoir une épouse, on les livrerait aux flammes. ${ }^{18}$

12 Erasme n'excuse pas l'1mmoralité ecclésiastique : * Reprehendi merentur qui pernicioso populi exemplo concubinas domi alunt". Cfr Allen, Opus eptstolarum, t. III, p. 373, 1. 437-438 (n० 858). - Voir aussi G. Chantraine, Mystere et Philosophie du Christ selon Erasme, p. 129, n. 131, Namur, 1971 .

13 De interdicto esu carnium, dans L.B. t. Ix. Col. 1201 F. Ce livre est de 122 comme le De conscribend Jean Fabri, evêque de vienne, se voit reprocher davoir pris la défense du célibat dans le but de ne pas perdre l'énorme profit des amendes infligées aux concublnaires. Cfr H. Jedin, Le Concile de Trente, t. I, p. 363, Paris, 1965.

p. 14 Allen, Opus, t. III, p. 377, 1. 609-611 (no 858). - G. Chantraine, op. cit., p. 131. n. 136. rexte reprodult dans $L . B .$, t. VI, col. 934 C, d'après les Annotationes de 1519.

16 Ibidem.

17 L.B., t. VI, col. 685 F. d'après les Annotationes de 1519.

18 Telle, Erasme et le septieme sacrement, p. 93. Cfr L.B., t. VI, col. 100 : : "In qua classe ponemus eorum genus qui vel arte vel metu detruduntur in coelibatum, ut scortari liceat, uxorem ducere non liceat : adeo ut si profiteantur concubinam sint catholici sacerdotes, sin uxorem dici malint conilciantur in Ignem. " - Il est à noter que ce passage manque aux Annotationes de 1519; il doit provenir d'une édition ultérieure, probablement celle de 1522 ou celle de 1527, que je n'ai pas pu identifier. 
Ces prises de position, - dues peut-être pour une part à l'influence de Jacques Lefèvre d'Etaples ${ }^{19}$, - ne passent pas inaperçues et le théologien espagnol Jacques Stunica, parmi d'autres, accuse leur auteur de mépriser la loi du célibat ecclésiastique. Erasme se défend en précisant sa thèse: a Je condamne ceux qui vivent dans le célibat d'une manière indigne en suivant leurs passions à la vue de tous. C'est ceux-là que j'estime ne pas devoir être admis au sacerdoce, à moins qu'ils ne puissent se racheter par le mariage. ${ }^{20}$

Jusqu'ici l'argumentation d'Erasme se borne à critiquer une tradition ecclésiastique parce qu'elle est trop exigeante pour pouvoir être observée sans susciter d'énormes problèmes. Il demande donc l'assouplissement de la loi du célibat: plutôt le mariage que le concubinage ! C'est là une irréfutable considération de fait, mais la motivation théologique fait défaut à ce point de vue moral, comme ses adversaires ne manquent pas de le souligner.

Erasme trouve la justification adéquate dans sa lutte contre le caractère monolithique et intangible des constitutions humaines de l'Eglise. En 1522, il écrit un livre sur ce sujet, le De interdicto esu carnium deque similibus hominum constitutionibus, qui conteste les traditions ecclésiastiques en général ${ }^{21}$. Par a approximations successives D, il décrit sa position touchant l'autorité de l'Eglise en matière de législation disciplinaire. a $A$ ses yeux, une telle législation n'a de valeur définitive et absolue que dans la mesure où elle répète les normes de l'Evangile. ${ }^{22} \mathrm{Si}$ elle est trop rigoureuse, elle devient inhumaine. On ne trahirait pas la pensée d'Erasme en avançant que, pour lui, ce qui n'est pas humain n'est pas chrétien ${ }^{23}$.

Tout en redisant son respect pour les coutumes et usages reçus des Apôtres, Erasme discute a l'opportunité de maintenir certaines lois ecclésiastiques comme le jeûne, l'abstinence, les jours de fête (excepté le dimanche et quelques fêtes du Seigneur), l'obligation du célibat pour les prêtres, etc. Ces institutions, introduites pour favoriser la piété, sont

19 Telle, Erasme et le septième sacrement, p. 239. - Massaut, Josse Clichtove, t. II, p. 182. - L'influence de Valla est vraisemblable : Telle, op. cit., p. 252: M. Fols, Il pensiero cristiano di Lorenzo Valla, p. 286 , Rome, 1969. - Sur d'autres docteurs favorables à la même thèse, voir Telle, Dilutio, p. 83, $\mathrm{n}$. 85 ; - Erasme

et le septieme sacrement, $\mathrm{p}$. 189 , n. 1 . 364 A. - A la même époque, 1521, un prédicateur de Bruges croit devoir prendre contre Erasme la défense du célibat: Allen, Opus, t. IV, p. 572, 1. 29-32 (n 1231 ). - Déjà en 1519, frasme avait répondu aux critiques adressées à l'Encomium matrimonii par Jean Briard, de l'université de Louvaln: Apologia pro declamatione matrimonii, dans L.B., t. IX. col. 105-111.

21 Erasme est à Bale depuis la fin de 1521. I 7 publiera la majeure partie de son œuvre. In n'y a aucune raison de penser qu'trasme a ecrit ce livre parce qu'il aurait été "mis en demeure de donner son avis sur le scandale des mariages dans les rangs du clerge " : Telle, op. cit., p. 192.

22 J. Coppens, Scholia, dans Scrinium Erasmianum, t. II, D. 599, Leyde, 1969. - J. Tracy, Erasmus, p. 148, Genève, 1972.

23 Voir, entre autres: Encomium matrimonii, dans A.S.D., t. I-5, p. 400, 1. 198-200. Ici-méme. note 27. 
souvent un empêchement pour la pratique d'une vraie piété chrétienne, qui doit être libre des “ cérémonies judaĩques ${ }^{24}$ et des superstitions. Erasme propose clairement qu'on bannisse ces lois ou, au moins, qu'on n'en exige pas l'observance afin que des institutions humaines n'entravent pas la liberté des chrétiens. Un respect, une considération excessive à l'égard des cérémonies et des pratiques religieuses peuvent nuire au véritable esprit évangélique, car le chrétien peut être amené à mettre trop de confiance dans les pratiques extérieures. $\searrow^{25}$

Après tout, pourquoi l'Eglise serait-elle seule à ne pas évoluer ? L'assistance de l'Esprit n'est pas une garantie d'immobilisme; au contraire, elle constitue une promesse de créativité continue. Ce que 1'Eglise a fait, elle peut le défaire, et Jésus ne lui a pas légué l'exemple d'un étroit conformisme: In vanum autem me colunt, docentes doctrinas et praecepta hominum ${ }^{26}$.

Erasme, dans le De interdicto, insiste sur l'absence de toute loi du célibat venant du Christ ou des Apôtres ${ }^{27}$. Il poursuit en montrant que la continence n'est pas essentielle au sacerdoce et que la discipline s'est adaptée aux circonstances historiques. « Jadis, ajoute-t-il, l'Eglise abolit la pratique des veilles nocturnes aux tombeaux des martyrs, même si la coutume générale des chrétiens les avait admises depuis plusieurs siècles. Quant au jeûne, qu'on prolongeait habituellement jusqu'au soir, l'Eglise en a ramené la limite à midi. Elle modifia beaucoup d'autres coutumes encore au fur et à mesure que les circonstances l'exigeaient. Pourquoi nous attacher ici avec une telle obstination à une institution humaine [le célibat sacerdotal], surtout quand il y a tant de raisons pour nous convaincre de la nécessité d'un changement? Tout d'abord, en effet, quantité de prêtres mènent une existence entachée par la mauvaise réputation et célèbrent les saints mystères avec une conscience peu tranquille. Ensuite, leur œuvre perd une grande partie de son fruit parce que leur vie honteuse inspire au peuple le mépris de leur enseignement. Or, si l'on permettait aux incontinents de se marier, non seulement ils connaîtraient une vie plus tranquille, mais encore ils retrouveraient leur autorité pour prêcher au peuple la parole de Dieu ; en outre, ils prendraient soin de donner à leurs enfants une éducation libérale et, firalement, ils cesseraient d'être les uns pour les autres un sujet de honte. Je ne dis pas cela pour me faire le répon-

24 Entendez : les pratiques religieuses et morales comparables a celles que Jésus reprochait aux pharisiens.

$25 \mathrm{~J}$. M. de Bu anda, Erasme de Rotterdam. Liberté et unité dans l'Église, p. 19-20. Sherbrooke, 1971. - Cet ouvrage donne aussi la traduction francaise du De interdicto esu carnium. - Texte latin dans L.B. t. IX. col. 1197-1214.

26 Marc, VII. 7. col. 1201 C. Dans l'Exomologesis, 1524, Hrasme ecrira: « Christus non exegit a quoquam coelibatum ». Cfr L.B., t. V, col. 155 C. D Dans ses Annotationes de 1519 , à propos de I. Cor., vII, 39, il écrit : "Christus virginitatem non exigit, ne videatur cum natura pugnare, licet beatos pronunciet qui hoc possint capere, sed addit : propter regnum Del. Cfr L.B., t. VI, col. 695 D. Volr Telle, op. cit., p. 190, 238. 
dant ou l'avocat des prêties qui, récemment, sans l'autorisation des papes, se sont mis à prendre femme ${ }^{28}$, mais je veux inviter les princes de l'Eglise à s'interroger sur l'opportunité des mesures qui permettraient d'adapter une législation antique aux intérêts du présent. En attendant, je voudrais aussi mettre en garde les évêques contre la témérité avec laquelle, sans avoir fail d'enquête, ils admettent n'importe qui dans les ordres. ${ }^{29}$

En effet, les candidats au sacerdoce doivent être interrogés avec soin par leurs supérieurs. Ils doivent surtout s'interroger eux-mêmes et scruter leur conscience afin qu'ils ne s'engagent point par esprit de lucre ou de facilite ${ }^{30}$.

Enfin, non sans malice, Erasme avertit les prêtres qui penseraient trouver un refuge dans le mariage d'y regarder à deux fois, a de crainte qu'une double pénitence ne vienne les torturer, celle du sacerdoce et celle du mariage : duplici poenitentia discrucientur, et sacerdotii et coniugii $^{31}$.

Désormais, la doctrine d'Erasme sur le célibat sacerdotal est, à quelques détails près, complète et cohérente. Il n'en changera pas. II restera sur ses positions et il les défendra contre vents et marées.

L'explosion de la Réforme rend toutefois sa thèse plus difficile à justifier devant l'opinion catholique offusquée par le mariage des prêtres dissidents ${ }^{32}$. En effet, il ne s'agit pas là d'un assouplissement de la règle canonique, comme le souhaitait Erasme, mais d'une manifestation de la liberté conquise aux dépens de Rome ${ }^{33}$. On se tromperait grossièrement en croyant qu'Erasme s'en réjouit ${ }^{34}$, car c'est de l'Eglise et non des individus qu'il espère l'aménagement du statut sacerdotal. En critiquant ces unions, il ne les déclare pourtant pas nulles, mais il déplore leur conclusion suspecte ${ }^{35}$.

Il se défend d'être pour quelque chose dans ces mariages, sans

28 C'est ce que M. Telle (op. cit., p. 192) appelle une « épldémie de marlages de prêtres et de moines suscitée par l'Encomium ".

29 L.B., t. IX, col. 1201 C-D. - Traduction de R. Galtbols dans J.M. de Bujanda, op. cit., p. $39-40$

30 Crasmé revient volontiers sur les qualités nécessaires aux prêtres et sur les défauts qui les menacent : Allen, Opus, t. VIII, p. 381, 1. 147-160 (no 2284).

31 L.B., t. IX, col. 1201 E.

32 Gocieanus exprime le sentiment général des catholiques lorsqu'll écrit à crasme en 1528 : " postquam Luterus eius sacri praesul factus est, cepit inter istos propemodum esse articulus fidei, sine uxore neminem esse christianum 》. Cfr Allen, Opus, t. VII, p. 517-518, 1. 48-50 (n० 2063).

33 En 1521, Capiton signale des mariages de prêtres à Halle. Cir Allen, Opus, t. IV, p. 597, 1. 26-28 (no 1241). - En 1522, le carme strasbourgeols Tilman de Lyn déciare légitime le mariage des prêtres. Le curé Matthieu Zell, de la nıême ville, se marie l'année suivante. Cfr M. Lienhard et J. Rott, Die Anfänge der evangelischen Predigt in Strassburg, dans l'ouvrage collectif Bucer und seine Zeit, p. 69, Wiesbaden, 1976. - On sait que Luther et Zwingli ne pensaient pas autrement.

34 Telle, Erasme et le septième sacrement, p. $330,336$.

34 Telle, Erasme et le septieme sacrement, p. 330, 336. Opus, t. VI, D. 402, 1. 81-83 (no 1744). - Apologia brevis ad Albertum Pium, dans L.B., t. IX, col. 1187 A. - Volr aussi Franzen, op. cit., D. 46. 
que l'on puisse savoir si la lecture de ses œuvres a pu influencer sur ce point l'un ou l'autre dissident ${ }^{36}$. Erasme ne comprend pas que, pour les prêtres passés à la Réforme, le mariage est un moyen de rompre avec le système et de sortir de l'ancien clergé. Sacerdotes multi pro laicis se gerunt, déclare-t-il avec indignation ${ }^{37}$. Il s'étonne, ou feint de s'étonner, que les nouveaux prophètes ne résistent pas mieux aux entraînements de la chair. Le nouvel Evangile serait-il inséparable de ces nouveaux ménages ?...

Erasme signale en passant le mariage de Juste Jonas et celui d'André Carlstadt ${ }^{38}$. En 1524, son correspondant Gaspard Hédion se marie et la nouvelle émeut et occupe Erasme davantage. Sa première réaction est franchement mauvaise et plutôt méchante. Il écrit à Willibald Pirckheimer : Hedio noster penultimo maii duxit uxorem Argentorati bene dotatam $^{39}$. Quelques jours plus tard, il félicite Hédion en une phrase rapide: Precor ut novum matrimonium vobis sit felix. Il pouvait difficilement être plus bref et moins chaleureux. Après quelques digressions, il poursuit par un rappel très ferme de ses opinions : " Jadis, pour l'amour de l'Evangile, ceux qui avaient une femme la quittaient de plein gré. Aujourd'hui, l'Evangile fleurit chaque fois que l'un ou l'autre épouse une femme richement dotée ${ }^{40}$. Je ne dis pas cela parce que je désapprouverais sans réserve le mariage des prêtres. On pourrait l'admettre s'il était conclu par nécessité, avec la permission des supérieurs, sans rébellion et d'un cœur sincère. Aujourd'hui, je crains que beaucoup ne se marient pour la seule raison que le mariage leur est interdit. Je sais combien est scandaleux le célibat de certains, mais le mariage favoriserait-il toujours la chasteté ? Que dire si un prêtre, qui hier avait une concubine, a aujourd'hui une femme et une maîtresse ? Et cependant, devant tous les papistes, [...] j'ai toujours déclaré franchement qu'il ne fallait pas refuser tout uniment le mariage aux candidats à la prêtrise s'ils ne pouvaient garder la continence. Je ne m'exprimerais pas autre-

36 Grasme écrira en 1526 : "Neque quisquam sacerdos nec ulla sacra virgo nupsit me auctore, nonnulios in suo instituto confirmavi ". Cfr Supputationes, dans I..B., t. IX, col. 589 A. - Et en 1532 : "Quid 1gitur frontis habent qui clamant me autore ista fieri quae fieri videmus? "Cfr J. Coppens, Scholia, dans Scrinium Erasmianum, t. II, p. 606.

37 Allen, Opus, t. VII, p. 19, 1. 218-219 (n० 1805).

38 Allen, Opus, t. v, p. 15, 1. 7-8 (n० 1258), lettre du 9 février 1522. - Le 21 fuillet 1524, erasme écrit sans commentaire : "In Hollandia mea passim fugiunt monachae ac nubunt in Domino ». Cfr Allen, Opus, t. V, p. 494, 1. 12-13 (no 1466$)$. 39 Allen, Opus, t. V, p. 469, 1. 20-21 (n० 1452).

40 Phrase doublement ironique. D'une part «Floret Evangelium est une expression qu'erasme réserve aux évangéliques. Voir, entre autres, l'Epistola ad pseudoevangelicos, dans L.B., t. $X$, col. 1579 D-E : "Novam vero libertatem evangelicam, impune facere sentireque quod cuique libitum [...] Nunc floret evangelium quod sacerdotes et monachi, contra leges certe humanas, contra professionem suam, ducunt uxores 》. Cfr N. Peremans, Erasme et Bucer, p. 67-89, Paris, 1970. L'expression ne peut donc être entendue à la lettre comme si frasme se rejoulssait "de voir la réalisation des souhaits exprimés dans la Declamatio matrimonii »: Telle, op. cit., p. 336 et 448. - D'autre part, parml les u uxores bene dotatas " figure la femme de Hédion, comme on l'a vu plus haut. 
ment devant le Souverain Pontife, non que je ne préfère la continence ${ }^{41}$, mais parce que je n'en vois que quelques-uns qui l'observent. Par ailleurs, pourquoi a-t-on besoin d'un si grand nombre de prêtres? Pour ma part, je n'ai pas poussé un seul prêtre vers le mariage, mais je n'en ai voulu à aucun parce qu'il désirait se marier. ${ }^{42}$

Cette lettre traduit clairement les réactions d'Erasme devant le mariage des chefs de la Réforme. Ses sentiments ne sont pas moins vifs, mais la moquerie est plus apparente lorsqu'il apprend le mariage de Capiton ${ }^{43}$, de Luther ${ }^{44}$ ou d'CEcolampade ${ }^{45}$. Il est particulièrement sarcastique pour ce dernier: Ante pauculos dies CEcolampadius duxit uxorem, puellam non inelegantem, cupiens in quadragesima macerare carnem ${ }^{46}$.

Après les tribuns de la Réforme, leurs seconds suivent le mouvement et ils échangent les liens du sacerdoce contre ceux du mariage. Leur nombre impressionne Erasme qui leur refuse estime et indulgence. On dirait qu'il pressent le tort que feront à ses idées ces unions insolites. Ce n'est pas ainsi qu'il avait envisagé l'émancipation des prêtres. De là une mauvaise humeur qui est compréhensible et une agressivité qui nous gêne.

Il écrit à Spalatin : "Ils abandonnent leur habit et prennent femme ; ensuite, ils justifient leur conduite ; entre-temps, ils sont à l'affût d'une situation brillante et sûre. " ${ }^{47}$ A Pirckheimer : "Ils ne cherchent que deux choses, l'argent et le mariage. " ${ }^{48}$ Enfin, dans ses Scholia: "Au défi de toute autorité, ils se marient. Ils ne se contentent pas d'exhorter les autres à faire de même : ils les y obligent, comme s'il était impie de ne pas se marier. Combien grande est l'insolence de ceux qui me rendent 'responsable de cet état de choses ! ${ }^{49}$

Au début de 1525, Erasme envoie au Conseil de la ville de Bâle

41 Erasme n'a jamais cherché à se libérer du célibat. On peut croire qu'il pense a son propre cas lorsqu'il ecrit : "Nihll magis optandum quam ut sacerdos immunis a coniugio, liber ac totus serviat Domino suo". Cfr De interdicto esu, (fausse) du mariage d'trasme en 1531, celui-ci ne fit qu'en rire: Allen, Opus, t. IX, p. 285, 1. 14-22 (n० 2508), p. 314, 1. 21-25 (n。2518), p. 340, 1. 19-24 (n०2534). 42 Allen, Opus, t. V, p. 482-483, l. 86-101 (nu i459).

43 Allen, Opus, t. V; p. 529, 1. 16-17 (no 1482). 44. 240, 1. 6-10 (n० 1653), p. 242, 1. 1-3 (no 1655), p. 283, 1. 1-6 (no 1677); t. IX, p. 456, i. $481-482\left(n^{\circ} 2615\right)$.

45 Allen, Opus, t. VII, p. 369, 1. 9-12 (n० 1979), p. 503, 1. 27-28 (n० 2054).

46 Allen, Opus, t. VII, p. 367-368, 1. 71-73 (no 1977). - La lettre est ecrite du-

rant le carême. - Cfr K. H. Oelrich, Der späte Erasmus, p. 27, Munster, 1961.

47 Allea, Opus, t. v, p. 551, 1. 8-10 (no 1497).

48 Allen, Opus, t. VII, D. 366, 1. 42 (no 1977). appréclations plus ou moins caustiques d'erasme : Allen, opus, t. V, D. $578,1$. 15-20 (no 1514); t. VI, p. 25, 1. 13-15 (no 1548); t. VII, p. 189. 1. 15-18 (n० 1883), p. 199-200, 1. 17-19 (no 1887), p. 360, l. 14-15 (no 1973), p. 543, 1. 47-49 (no 2079): t. IX, p. 457, 1. 486-488 (n० 2615 ). - Notons que, en 1531, le magistrat protestant de Strasbourg reproche à certains prêtres mariés de "ne se prévaloir des doctrines nouvelles que pour justifier leur mariage ». Cfr Fr. Wendel, L'Eglise de Strasbourg. p. 32. Paris, 1942. 
une longue lettre sur la conduite à tenir devant les difficultés religieuses de l'heure ${ }^{\text {so. }}$. Il s'agit là d'un document soigneusement mûri, proposant une opinion nuancée. Sur la question du célibat sacerdotal, l'avis d'Erasme s'exprime en huit points dont voici l'essentiel :

$1^{\circ}$ Aucune recommandation ne serait justifiée en faveur des prêtres qui se marient temere, c'est-à-dire sans autorisation (p. 10, 1. 116-117).

$2^{\circ}$ Les supérieurs pourront prendre en considération le cas de ceux qui sont entrés par erreur dans la vie ecclésiastique (1. 117-120).

$3^{\circ}$ Ils devront être sévères pour ceux qui excitent les autres, quasi per se sit impurus sacerdotum coelibatus (1. 120-125).

$4^{\circ}$ Une très grande prudence s'impose à l'encontre des prêtres mariés, alors qu'ils sont connus pour avoir eu plusieurs concubines, metuendum ne impuro coelibatui succedat impurius matrimonium (1. 126-134).

$5^{\circ}$ La règle générale subsiste autant que possible : mieux vaut un prêtre marié qu'un prêtre concubinaire (1. 135-143).

$6^{\circ}$ S'il s'agit de prêtres ignorants et indignes, il est souhaitable qu'ils puissent aussi se marier, mais qu'ils renoncent à l'exercice de leur sacerdoce et vivent en laïcs, deponerent sacerdotium ac pro laicis haberentur (1. 143-144).

$7^{\circ}$ Quant aux prêtres instruits qui, de bonne foi, ont reçu les ordres et peuvent être encore utiles à l'Eglise, bien qu'ils soient incapables de respecter la continence, alors que pour le reste ils vivent correctement, ils pourraient se marier en conservant leurs fonctions, his concederem uxorem, nec adimerem sacerdotium (1. 145-150).

$8^{\circ}$ Enfin, la vigilance s'impose pour que les indignes ne puissent abuser des mesures qui précèdent (1. 151-159).

A travers cette véritable consultation, on voit Erasme comme pris de vitesse et dépassé sur sa gauche par les dissidents. Néanmoins, les principes demeurent, même s'ils deviennent chaque jour d'une application plus malaisée.

Sur sa droite, il n'est pas plus rassuré. Rome ne bouge pas ! Bien qu'Erasme se soit déclaré publiquement contre Luther, il n'a pu désarmer la Sorbonne qui lui reproche les thèses du De interdicto esu carnium. Noël Béda, le théologien le plus sourcilleux de Paris, énumère ses griefs. Le premier est d'avoir mal parlé du célibat ecclésiastique : plurima perniciose et in grave populi christiani scandalum, utpote de cleri coelibatu ${ }^{51}$.

Erasme réplique par une pirouette en rappelant qu'il a écrit aussi

50 Allen, Opus, t. VI, p. 6-11 (n• 1539). La lettre a été imprimée dès 1526 sous le titre Consilium Erasmi Roterodami in causa evangelica. - Cfr Telle, op. cit., p. 199 . Allen, Opus, t. VI, p. 83, 1. 90-92 (n॰ 1579 ). 
une apologie du célibat : Extat de coelibatu apologia ${ }^{52}$. En fait, il ose baptiser de ce nom la courte Epistola dissuasoria qui complète l'Encomium matrimonii dans le De conscribendis epistolis ${ }^{53}$. Puis, pressé dans ses derniers retranchements, il loue le célibat, tout en ajoutant que la situation actuelle du clergé, surtout en Allemagne, l'incline vers le mariage des prêtres: vehementer laudo coelibatum, sed ut nunc se habet sacerdotum ac monachorum vita, praesertim apud Germanos, praestaret indulgeri remedium matrimonii ${ }^{54}$.

La dispute continue et Béda rassemble ses arguments en un volume d'Annotationes auquel Erasme répond, en la même année 1526, par ses Supputationes ${ }^{55}$. Il y affirme que le célibat ecclésiastique n'est pas de droit divin (ce qui aurait annulé tous ses efforts) et que, par consequent, le pape ou le concile ont le pouvoir d'en dispenser. Le mariage des prêtres est un moindre mal, levius malum, mais c'est un remède approprié à la situation, faute de mieux. Sur un point, Erasme opère une retraite peu glorieuse. Il se serait, dit-il, opposé aux clercs qui prennent prétexte de leur faiblesse pour ne pas lutter contre elle: opponitur eis qui praetextunt incontinentiam, cum non dederint operam ut possint esse continentes ${ }^{56}$.

Néanmoins, Béda considère Erasme comme un dangereux contestataire et il le classe parmi les crypto-luthériens (lutherani clandestini) ${ }^{57}$. Josse Clichtove, théologien français d'origine flamande, ne conclut pas autrement son Propugnaculum Ecclesiae (1526). Pour ce champion du sacerdoce, le célibat ecclésiastique est de droit divin. Dès lors, la continence des prêtres revêt l'obligation irrévocable d'un vœu solennel ${ }^{58}$. Erasme n'en croit rien. Il maintient le caractère relatif de la discipline et il répond à ses adversaires dans l'Institutio christiani matrimonii, qui est aussi de 1526, puis dans ses Declarationes, sa Dilutio ${ }^{59}$ et ses Scholia, tous trois de 1532. Il rappelle que l'Eglise orientale permet la prêtrise aux hommes mariés alors que, dans l'Eglise latine, les minorés doivent renoncer à leurs bénéfices pour garder leurs femmes ${ }^{60}$.

52 Allen, Opus, t. VI, p. 105, 1. 732 (no 1581). - Erasme aurait pu citer. a plus juste titre, la Comparatio Virginis et Martyris qu'll avalt publiee en 1523: L.B., t. V. col. 589-600.

53 A.S.D., t. I - 2, o. 429-432.

54 Allen, Opus, t. VI. p. 182, 1. 50-52 (n० 1620).

55 Appelées aussi Divinationes et Apologia.

56 L.B., t. IX, col. 488 F - 489 A. A Erasme reviendra sur le dernier point dans son Appendix de scriptis Clithovei (L.B., t. IX, col. 812 F). 57 En 1531 . Eustache de Zichem accusera erasme de penser aussi mal que
Luther du célibat ecclésiastique. - Cfr Eustachius de Zichinis. Erasmi canonis quinti interpretatio (ed. J. Coppens), p. 35, Bruxelles, 1975.

58 J.-P. Massaut, Le célibat dans l'idéal sacerdotal de Josse Clichtove, dans Sacerdoce et celibat (ed. J. Coppens), p. 459-506, Louvain, 1971. - J. Coppens, Erasme et le célibat, dans le mêne volume, $0.452-458$. - sur la questin in vau solennel, voir Apologia brevis ad Albertum Pium (1531), dans L.B., t. IX, col. 1183 D - 1185 B. - Cfr Telle, op. cit., g. 142, 399.

59 Telle (Dilutio, p. 59) a remarqué avec pertinence que la Dilutio manque aux Omnia opera d'erasme publiés à Bale en 1540. Il nous parait stgnificatif que l'Encomium matrimonii solt dans le même cas. - sur les Declarationes et les Scholia, voir plus loln. La Dilutio a eté tradulte par P. Mesnard, Erasme. La philosophie chrétienne, Paris, 1970.

60 Institutio christiani matrimoni, dans L.B., t. V, col. $637 \mathrm{C}$. 
Elevant le débat, Erasme revient à la charge, répétant que le mariage est un sacrement et que la virginité ne l'est pas ${ }^{61}$. De plus, le célibat n'est pas la virginité ${ }^{62}$ : ils sont aussi différents l'un de l'autre que peuvent l'être une catégorie juridique et une option morale. Les prêtres ne cultivent pas tous l'esprit de virginité ; de même, si l'on ose ainsi dire, les prostituées célibataires ne passent pas pour des vierges.

Si Erasme reconnaît en principe la supériorité de la virginité sur le mariage, toute son œuvre respire le sentiment contraire ${ }^{63}$. La virginité, écrit-il, est a divine $»$, « céleste , “ angélique ». C'est pour lui un idéal malaisément accessible au commun des mortels ${ }^{64}$, et dont il faut détourner les candidats trop jeunes, incapables de mesurer les risques de leur engagement. Par ailleurs, Erasme ne conseille pas d'ordonner des hommes mariés, car, au début du $\mathrm{XVI}^{\mathrm{e}}$ siècle, cette question ne se pose plus, ou pas encore... Rien non plus sur le sacerdoce féminin !

On a cru parfois que la Diète d'Augsbourg, en 1530, a été l'occasion pour Erasme de rompre une lance en faveur du mariage des prêtres comme en faveur de la communion sous les deux espèces ${ }^{65}$. En réalité, Erasme, qui n'est pas allé à Augsbourg, reste en contact épistolaire avec plusieurs amis présents à la Diète, et avec Charles-Quint luimême ${ }^{66}$. L'assemblée, réunie dans un esprit de conciliation très érasmien, décevra pourtant les espoirs de tous.

En 1531, la Sorbonne publie enfin l'ensemble de son dossier antiérasmien : Determinatio Facultatis theologiae in schola Parisiensi super quam plurimis assertionibus D. Erasmi Roterodami. Erasme reproduit ces censures, pour mieux les réfuter, dans ses Declarationes ad censuras Lutetiae vulgatas, publiées à Bâle en janvier-février de l'année suivante $^{67}$.

61 Institutio, dans L.B., t. V, col. 620 A. - Telle, Dilutio, p. 90.

62 II l'affirmait dêjà dans l'Apologia pro declamatione matrimonit, dans L.B., t. IX, col. 109 A. I Petit débat phllologique a ce propos entre trasme et Ambroise Pelargus : Ailen, Opus, t. $X, p 47,1.1-12$ (n० 2674), p. 48, 1. 1-4 (n० 2675 ), p. 49, 1. 1-14 (n० 2676). En 1524, falsant allusion a la mort de la reine de France, rrasme évoqualt Francols Ier « cul coellbatum accessisse doleo ». Cfr Allen, Opus, t. v. D. 531, 1. 15 (no 1484).

63 Apologia adversus monachos quosdam Hispanos, dans L.B., t. IX, col. 1089 E-F. Apologia ad Albertum Pium, dans L.B., t. IX, col. 1187"A.

64 Dejà dans l'Encomium matrimonii de 1618 : A.S.D., t. I - 5, D. 404, 414. Volr aussi Virginis et martyris comparatio, dans L.B., t. $\dot{v}$, col. 596 B; - Allen, Opus, t. VI, p. 402 , 1. 86 (no 1744) ; - L.B., t. III a, col. 779 F. - En 1529. Erasme se moque de la théologie regnante quí considère que la continence est une chose très facile qui ne dépend que de la volonté : * Discimus continentiam esse rem facillimam, modo velit homo 》. Cfr Allen, Opus, t. VIII, p. 195, 1. 19-20 (n 2178). Voir aussi Telle, Dilutio, p. 82.

65 Franzen, op. cit. p. 42-46. - Coppens, op. cit., p. 454, n. 87. - P. Rassow, Die politische Welt Karls $V$., p. 40, 54, 60, 63, Muntch, 1946. - Oelrich, op. cit., p. 22. On sait, d'autre part, que la Confession d'Augsbourg ecartait la lol du célibat sacerdotal.

66 La lettre d'erasme a Charles-Quint, cltée par Melanchthon dans sa lettre du 27 julllet a Luther, est perdue. Cfr Luthers Werke. Briefwechsel, t. v, p. 508, Weimar, 1935. - Voir aussi Allen, Opus, t. IX, p. 157, 1.16-17 (no 2442) ; p. $321,1$. 143-158 (n० 2522). - Jedin, Le Concile de Trente, t. I. p. 227-237.

143 Le 4 févier 1532, il envole le volume a Plerre Tomicki: Allen, Opus, t. IX, p. 419, 1. 24 (n० 2600). 
La Sorbonne ne fait grâce à Erasme d'aucune critique. A côté de ses ouvrages théologiques, les Colloques sont soumis à une lecture sévère. C'est ainsi que, à propos du dialogue Proci et puellae, il est reproché à son auteur de faire un éloge excessif du mariage et d'enseigner le mépris de la virginité. Mieux encore, le dialogue affirmerait que la virginité s'apprend per actus carnales! Erasme se justifie en rappelant que les interlocuteurs des colloques expriment chacun leur opinion et qu'il leur en laisse la responsabilité ${ }^{68}$.

La Dilutio eorum quae Iodocus Clithoveus scripsit adversus declamationem Des. Erasmi Roterodami suasoriam matrimonii est, comme son titre le proclame, une réponse à Clichtove et une apologie de l'Encomium matrimonii, publié quatorze ans plus tôt. Erasme ne retire rien des thèses de sa declamatio. Il les répète en les assortissant de quelques propos apaisants et bien dans sa manière : " Je crois la virginité préférable au mariage, si du moins elie est volontairement choisie par amour de la piété. " ${ }^{69}$ Et encore : " Je préfère le mariage au célibat, mais non à la continence. ${ }^{70} \mathrm{Et}$ enfin : " Ni le célibat, ni la virginité, ni la continence ne méritent d'être loués s'ils ne rendent pas l'homme plus libre de s'adonner à la piété. ${ }^{71}$

Cela dit, et adroitement dit, Erasme revient à ses grandes affirmations, mais les a-t-il jamais abandonnées ?... Son éloge du célibat ecclésiastique est d'autant plus grand que cet état ne convient qu'à une élite: "Il est fait pour les apôtres et pour les hommes apostoliques, ut rem perfectam perfectis. ${ }^{72} \mathrm{Et}$ pour les autres, qui ne sont point parfaits? Oubliant les précisions limitatives qu'il avait cru devoir glisser dans l'Institutio christiani matrimonii ${ }^{73}$, Erasme donne la réponse qui lui est familière : "A ceux qui ne peuvent garder la continence, qu'il soit permis d'épouser leurs concubines. ${ }^{74}$

Toujours en 1532, Erasme veut réfuter les objections faites à sa fameuse lettre $\mathrm{De}$ interdicto esu carnium, deque similibus hominum constitutionibus, publiée dix ans plus tôt. Il la réédite, non sans l'augmenter quelque peu ${ }^{75}$, et il la fait suivre de Scholia defensoria ${ }^{76}$, dans lesquels nous reconnaissons les thèmes qui lui sont chers: le célibat

68 Ce colloque (1l n'est pas le seul critiqué) est de 1523 : A.S.D., t. I - 3 , p. 277-288. - La réponse d'erasme dans L.B., t. IX, col. 937 C-F. . V Volr Telle, Erasme et le septieme sacrement, $p$. 300, 305, 3io. , Voir aussi F. Bierlaire, Les Collogues d'Erasme. Réforme des études, réforme des mœurs et réforme de l'Église au XVIe siècle, p. 245-246, 274-276, Liège et Paris, 1978. - Déjà en 1528, trasme répondait à des critiques semblables: Apolooia adversus monachos quosdam Hispanos, dans L.B., t. IX, col. 1085 F. 1089 E-F.

69 Telle, Dilutio, p. 70.

70 Dilutio, p. 76.

71 Dilutio, p. 81.

72 Dilutio, p. 82.

73 Voir plus haut, p. 507.

74 Dilutio, p. 84.

75 Dilutio, p. 60.

76 In Epistolam Des. Erasmi Roterodami de delectu ciborum ad Christophorum episcopum Basiliensem, scholia eiusdem defensoria (ed. J. Coppens). dans Scrinium Erasmianum, t. II, p. 599-620, Leyde, 1969. 
n'est pas de droit divin ; il ne peut être rompu que dans les conditions admises par l'autorité de l'Eglise; les prêtres ainsi libérés devraient compenser leur faiblesse par l'intégrité de leur vie et par de pieuses études; enfin et surtout, l'essentiel de la religion ne repose pas sur la messe, le jeûne et la continence, mais sur la foi, l'espérance et la charité ${ }^{77}$.

Erasme n'a plus que quelques années à vivre et il oriente ses derniers efforts dans d'autres directions. Désormais, il n'écrira plus sur le mariage et, par conséquent, sur le célibat. Il y fera cependant quelques allusions dans ses lettres ${ }^{78}$, dans l'Explanatio Symboli ${ }^{78}$ et, enfin, dans la grande cuvre de sa vieillesse, preuve ultime de son souci pastoral: l'Ecclesiastes sive de ratione concionandi ${ }^{80}$. La page est tournée et la question résolue, du moins en ce qui le concerne, car il n'a aucun pouvoir de décision. L'établissement souhaité d'une nouvelle discipline ecclésiastique, alliant l'orthodoxie et la tolérance, n'est pas de son ressort.

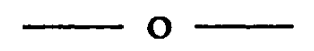

Si nous considérons dans leur ensemble les déclarations d'Erasme sur le célibat sacerdotal, nous pouvons en déduire qu'il a cherché sans cesse à apporter une solution correcte au problème du prêtre vivant dans le monde. Emu par la situation malheureuse de tant d'ecclésiastiques sans vocation ou sans continence, il a souhaité un adoucissement à la rigueur des lois sur le célibat, comme il a souhaité, pour des raisons comparables, l'adoucissement des lois sur l'indissolubilité du mariage ${ }^{81}$. Rien chez lui, par ailleurs, ne paraît annoncer l'idée de " déclergification 》 défendue par certains aujourd'hui.

Sa position est originale. Elle est modérée si on la compare à celle de Luther. Jamais Erasme n'a approuvé les unions conclues par des prêtres en rupture de ban. C'est à l'Eglise qu'il demande l'indulgence pour ceux qui ne peuvent plus tenir les promesses de leur ordination. D'une part, il admet le charisme du célibat virginal, signe d'une vocation authentique et forte. D'autre part, il voudrait que les prêtres légitimement mariés ne renoncent pas à leur rôle pastoral, à moins qu'ils

77 Scholia, nos 17, 18, 20, 22, 28

78 Particulièrement dans la correspondance échangée avec Ambrolse Pelargus ; voir plus haut, p. 508, n. 62. - Voir aussi une lettre de Christophe de Stadion : Allen, Opus, t. X, p. 191, 1. 53-69 (n ${ }^{\circ}$ 2787).

79 Dans ce livre de 1533, Erasme écrit : "Est quidem honorabile coniugium caste servatum, sed longe honorabilior virginitas perpetua, modo spontanea et pietatis amore suscepta». Cfr L.B., t. V, col. 1155 . Ce texte reproduit presque textuellement une affrmation de la Dilutio, op. cit., p. 70.

80 Ce livre est de 1535. Voir quelques allusions peu significatives dans $L . B$., t. V, col. 894 A, 904 E, 917 D. Cfr Telle, op. cit., D. 337 . F. - C1 Responsio ad disputationem Phimostomi (1532), dans L.B., t. IX, col. 961 
ne s'en montrent vraiment indignes ${ }^{82}$. Enfin, il n'a pas eu la naiveté de croire que le mariage supprimerait toutes les difficultés de la vie sacerdotale ; il lui a suffi d'espérer qu'il en aplanirait certaines et rapprocherait le prêtre des laïcs.

\section{$\mathbf{0}$}

Le scandale provoqué dans les milieux catholiques par le mariage des prêtres passés à la Réforme a réduit presque à néant les espoirs d'Erasme. Le Concile de Trente ne l'a pas suivi ${ }^{83}$, mais il n'a pas non plus adopté toutes les vues de Clichtove, qui prétendait alourdir encore le célibat en le déclarant lié à un vœu solennel ${ }^{84}$.

Aujourd'hui, on peut dire que les idées d'Erasme sur le célibat sacerdotal ont fait du chemin; on doit reconnaître qu'elles n'ont pas triomphé.

Liège.

L.éon-E. HaLkin.

\footnotetext{
82 Voir plus haut, p. 506, no 6. - Cfr A. Esmein, R. Genestal et J. Dauvillier, Le mariage en droit canonique, t. II, p. 15i-155, 386, 490-492, Paris, 1935.

83 Malgré les efforts de plusieurs théologiens qui reprendront les idées d'trasme sur ce point. Cfr Esmein, op. cit., t. II, p. 270. - Bien sâr les láes d'trasme sur le célibat ne sont pas restées sans influence, parmi les protestants comme parmi les catholiques. L'érasmisme survivra à erasme et l'on connait les opinions en ce domaine de Pflug, Cassander et Witzel, parmi d'autres. 84 Voir plus haut, p. 507.
} 